\title{
Determinants of rural practice: positive interaction between rural background and rural undergraduate training
}

\author{
The strong \\ positive \\ interaction \\ between rural \\ background \\ and rural \\ clinical school \\ exposure \\ ... could help \\ inform policy \\ changes
}

Srinivas Kondalsamy-

Chennakesavan

MBBS, MPH, FRSPH

Diann S Eley

$\mathrm{PhD}, \mathrm{MSc}, \mathrm{BSC}$

Geetha Ranmuthugala MBBS, PhD

Alan B Chate MBBS, FACRRM, FRACGP'

Maree R Toombs $\mathrm{PhD}$

Deepak Darshan MBBS, MASc, PhD

Geoffrey C Nicholson

PhD, FRACP, FRCP

1 University of Queensland Toowoomba, QLD.

2 University of Queensland Brisbane, QLD.

geoff.nicholson@ uq.edu.au

doi: 10.5694/mjal4.00236

Online first 12/01/15

Editorial p 5

Research p 46 n developed countries, including the United States, Canada and Australia, a fifth to a third of the population live in rural areas yet the number of medical practitioners employed per 100000 population in those areas is about half that of major cities. ${ }^{1-4}$ It has long been recognised that rural doctors are more likely to have a rural background and to have had some medical training (undergraduate or postgraduate) in rural areas, ${ }^{5-16}$ although the effect of undergraduate rural exposure has been questioned.${ }^{17}$ Other factors associated with rural practice include being single, having children, having a partner with a rural background, rural primary and secondary education, intention or desire to practise rurally, sex, age, having a bonded scholarship, and medical school attended. ${ }^{18-20}$

From the early 1990s, the Australian Government introduced national initiatives aimed at encouraging rural practice. This included funding medical schools to increase enrolment of students with a rural background and provide short-term undergraduate rural exposure..$^{21,22}$ In 2001, to increase the duration of rural exposure, universities began to establish rural clinical schools (RCSs); by 2008, 17 were operating. Participating universities are funded to train $25 \%$ of domestic students who are publicly funded (those have a Commonwealth-supported place) in a rural area, defined by Australian Standard Geographical Classification - Remoteness Area (ASGC-RA) categories ASGC-RA2 to ASGC-RA5. ${ }^{23}$

To track students through medical school and training, the Australian Government has supported a large prospective cohort study involving all Australian medical students - the Medical Schools Outcomes Database and Longitudinal Tracking Project. ${ }^{24}$ In the future, this study will generate robust data (currently, most

\section{Abstract}

Objective: To determine the role of rural background and years of rural clinical school training on subsequent rural clinical practice.

Design, setting and participants: Retrospective cohort study of University of Queensland (UQ) medical graduates who graduated during the period 2002-2011 (contacted via internet, telephone and mail, using information obtained from UQ, the Australian Health Practitioner Regulation Agency, and telephone directory and internet searches) who completed an online or hard copy questionnaire during the period December 2012 to October 2013.

Main outcome measure: Current clinical practice in a rural location.

Results: Of 1572 graduates to whom the questionnaire was sent, 754 $(48.0 \%)$ completed the questionnaire. Of the respondents, $236(31.3 \%)$ had a rural background and 276 (36.6\%) had attended the University of Queensland Rural Clinical School (UQRCS). Clinical practice location was rural for 18.8\% (90/478) of UQ metropolitan clinical school attendees and $41.7 \%(115 / 276)$ of UQRCS attendees $(P<0.001)$. In the multivariate model with main effects, independent predictors of rural practice were (OR [95\% $\mathrm{CI}])$ : UQRCS attendance for 1 year (1.84 [1.21-2.82]) or 2 years (2.71 [1.654.45]), rural background (2.30 [1.57-3.36]), partner with rural background (3.08 [1.96-4.84]), being single (1.98 [1.28-3.06]) and having a bonded scholarship (2.34 [1.37-3.98]). In the model with interaction between UQRCS attendance and rural background, independent predictors of rural practice were rural background and UQRCS attendance for 1 year (4.44 [2.38-8.29]) or 2 years (7.09 [3.57-14.10]), partner with rural background (3.14 [1.99-4.96]), being single (2.02 [1.30-3.12]) and bonded scholarship (2.27 [1.32-3.90]). The effects of rural background and UQRCS attendance were duration dependent.

Conclusions: This study strengthens evidence that, after adjusting for multiple confounders, a number of exposures are independent predictors of rural medical practice. The strong positive interaction between rural background and rural clinical school exposure, and the duration-dependent relationships, could help inform policy changes aimed at enhancing the efficacy of Australia's rural clinical school program.

participants are undergraduates or are in early postgraduate training).

The University of Queensland Rural Clinical School (UQRCS) was established in 2002. It is one of the largest RCSs in Australia and has teaching sites in four regional cities situated $130-650 \mathrm{~km}$ from Brisbane. The University of Queensland (UQ) School of Medicine also has eight metropolitan clinical schools (MCSs).

We aimed to quantify determinants of rural practice and interactions between them, particularly the role of rural background and years of RCS training, for UQ medical graduates. We hypothesised that attendance at UQRCS is an independent predictor of rural practice, after adjusting for confounders, and that a positive interaction between UQRCS attendance and rural background enhances the effect.

\section{Methods}

This study was part of the UQ Medical Graduates Cohort Study a retrospective cohort study of UQ medical graduates who graduated during the period 2002-2011. Lists of eligible participants (those who had been domestic students [ie, Australian citizens and permanent residents]) and their current contact details, if available, were obtained from UQ. If not available, current suburb and 
1 Location of current clinical practice for study participants $(n=754)$ by clinical school attended, and distribution of Queensland's general population*

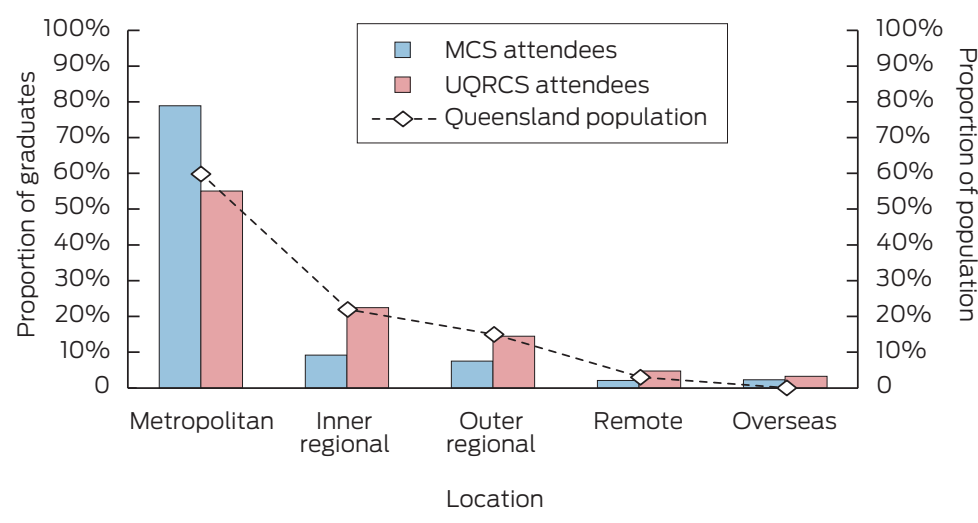

MCS = metropolitan clinical school. UQRCS = University of Queensland Rural Clinical School. * Distribution of Queensland population according to Australian Bureau of Statistics data for 2010 .

postcode were sought by searching the Australian Health Practitioner Regulation Agency (AHPRA) register of practitioners. This information was used to narrow telephone directory and internet searches. Potential participants were invited by email, post or telephone and sent a link to an online questionnaire, or a hard copy if requested. Since UQRCS attendance was a critical exposure variable but only $20 \%$ of potential participants would have attended UQRCS, we targeted this group to improve the power and efficiency of the study.

The questionnaire comprised questions on demographics, family information (parents' rural background, partnership status and partner's rural background), residential history (birth place and location during preschool, primary school, high school and post-school years), boarding school attendance, gap year after high school, scholarships (including bonded scholarships), rural health club membership, tertiary education and postgraduate training, year of medical school graduation, and location of current clinical practice (primary location for those with more than one). The outcome of interest was location of current clinical practice categorised as rural. The primary predictor variables of interest were attendance at UQRCS and rural background; the latter was defined as having resided in a rural area in Australia for at least 5 years since commencing primary school and before commencing UQ medical degree, as per funding parameters.

\section{Statistical analyses}

Descriptive statistics were used to report location of current clinical practice by ASGC-RA categories and UQRCS status. The category ASGCRA1 was considered metropolitan, and categories ASGC-RA2 to ASGCRA5 were considered rural (RA2, inner regional; RA3, outer regional; RA4, remote; RA5, very remote). If location of current clinical practice was overseas, it was categorised as metropolitan.

Univariate and multivariate logistic regression analyses were used to identify factors predictive of rural clinical practice. Multivariate models were adjusted for potential confounding factors: parent's rural background, partnership status, partner's rural background, bonded scholarship, boarding school attendance and gap year after high school. Interactions between these determinants were evaluated and included in the final model if statistically significant.

Stata for Mac (version 12.1, SE [StataCorp]) was used for statistical analyses and $P<0.05$ was considered statistically significant.

The UQ Behavioural and Social Sciences Ethical Review Committee approved the study.

\section{Results}

Of 2833 medical graduates who graduated during the period 20022011, 2478 were domestic students. Of these, contact information (email address, postal address or telephone number) was available for 1714 . Of these potentially contactable graduates, 142 were initially excluded emails bounced for 127 (no other contact details were available) and 15 declined to participate. The questionnaire was sent to the remaining 1572 , of whom 754 completed it during the period December 2012 to October 2013 (response rate, $48.0 \%$ [likely to be an underestimate as the status of sent emails could not be verified]). Characteristics of the participants are shown in Appendix 1.

Of the 754 respondents, $31.3 \%$ (236) had a rural background, 36.6\% (276) attended UQRCS for 1 or 2 years and $27.2 \%$ (205) were currently practising in a rural area - 18.8\% (90/478) of those who attended an MCS and $41.7 \%(115 / 276)$ of those who attended UQRCS $(P<0.001)$. Of those categorised as currently practising in a metropolitan area, 20 were practising overseas.

The proportion of participants currently practising in a rural area was lowest for those with a metropolitan background who attended a metropolitan clinical school (reference group; $16.9 \%$ [61/361]), intermediate for those with a rural background who attended a metropolitan school (24.8\% [29/117]) and for those with metropolitan background who attended UQRCS (26.8\% [42/157]), and highest for those with a rural background who attended UQRCS (61.3\% [73/119]). For all rural ASGC-RA categories, the proportion of practitioners who had attended UQRCS was about twice that of those who attended an MCS (RA2, 9.2\% [44/478] v 22.5\% [62/276]; RA3, 7.5\% [36/478] v 14.5\% [40/276]; RA4, 1.0\% [5/478] v $2.5 \%$ [7/276]; RA5, $1.0 \%$ [5/478] v $2.2 \%$ $[6 / 276])$. Also, the geographic distribution of UQRCS graduates matched the distribution of Queensland's general population more closely than that of MCS graduates (Box 1).

Associations between rural practice and potential predictors of rural 
2 Multivariate models predicting rural practice

\section{Odds ratio} $(95 \% \mathrm{Cl})$

\section{$P$}

Model with main effects
UQRCS attendance
MCS attendee
UQRCS attendee (1 year)
UQRCS attendee (2 years)
Background of participant
Metropolitan background
Rural background*
Background of partner
Metropolitan background
Rural background
Not applicable (single)
Bonded scholarship
No bonded scholarship
Bonded scholarship

Model with main effects

UQRCS attendance

MCS attendee

Reference group

$1.84(1.21-2.82)$

0.005

$2.71(1.65-4.45)<0.001$

Reference group

$2.30(1.57-3.36)<0.001$

Reference group

$3.08(1.96-4.84)<0.001$

$1.98(1.28-3.06) \quad 0.002$

Reference group

$2.34(1.37-3.98) \quad 0.002$

Model with interaction between UQRCS attendance and rural background ${ }^{\dagger}$

UQRCS attendance and background of participant

MCS attendee, metropolitan background

Reference group

MCS attendee, rural background

$1.61(0.94-2.75) \quad 0.08$

UQRCS attendee (1 year), metropolitan background

$1.46(0.85-2.51) \quad 0.17$

UQRCS attendee (1 year), rural background

$4.44(2.38-8.29)<0.001$

UQRCS attendee (2 years), metropolitan background

$1.83(0.91-3.67)$

0.09

UQRCS attendee (2 years), rural background

$7.09(3.57-14.10)<0.001$

Background of partner

Metropolitan background

Reference group

Rural background

$3.14(1.99-4.96)<0.001$

Not applicable (single)

$2.02(1.30-3.12)$

0.002

Bonded scholarship

No bonded scholarship

Reference group

Bonded scholarship

$2.27(1.32-3.90)$

0.003

UQRCS = University of Queensland Rural Clinical School. MCS = metropolitan clinical school. * Rural background was defined as at least 5 years since primary school and before commencing University of Queensland medical degree spent a rural area (areas in Australian Standard

Geographical Classification - Remoteness Area [ASGC-RA] categories ASGC-RA2 to ASGC-RA5) † Other two-way and three-way interactions (among UQRCS status, rural background status and partnership status) were not statistically significant and were not included in the model.

practice are shown in Appendix 2. On univariate analyses, the following variables showed an odds ratio (OR) of at least 2.0: UQRCS attendance; rural background; father's, mother's and partner's rural background; any scholarship; bonded scholarship; and rural health club membership. There was no association between sex and rural practice.

Two multivariate models predicting rural practice are shown in Box 2. In the model with main effects (without interaction terms), the following variables were independent predictors: UQRCS attendance (1 or 2 years); rural background; partner with rural background; being single; and bonded scholarship. With the exception of the interaction between UQRCS attendance and rural background, all other two-way and three-way interactions between UQRCS exposure, rural background and partner's rural background were not statistically significant and were not included in the final multivariate model. The variables relating to rural background exhibited multicollinearity, so parents' backgrounds were not included in the final multivariate model.

To simplify the interpretation of interaction between UQRCS attendance and rural background, the participants were grouped into six categories. The model that included interaction between UQRCS attendance and rural background shows that a substantial positive interaction exists (Box 2). Compared with the reference group, participants with a rural background who attended UQRCS for 1 and 2 years were 4.44 and 7.09 times as likely, respectively, to practise in a rural area after adjusting for partner with a rural background, being single and bonded scholarship.

To explore the effect of the duration of rural background on the adjusted predictive probability of current rural practice, we developed a logistic regression model with explanatory variables: UQRCS attendance $\mathrm{v}$ MCS attendance, years spent in a rural area (since primary school and before commencing UQ medical degree) as a continuous variable, and an interaction term between these two variables (Box 3 ). The predicted probabilities are divergent across the range of years spent in a rural area. In UQRCS attendees with 10 and 20 years of rural exposure, the predicted probabilities of rural practice are $54 \%$ (95\% CI, 46\%-62\%) and 79\% (95\% CI, $69 \%-89 \%$ ), respectively.

To determine the representativeness of our sample, we linked 2360 of the 2478 domestic medical graduates (95.2\%) to the AHPRA database to determine current practice location. Characteristics of these two groups and crude ORs of rural practice for them are shown in Appendix 3. All characteristics for these two groups are similar except there was a higher proportion who attended UQRCS in the group of 2478 students from which we recruited participants than the group of 2360 for whom data linkage was done (36.6\% v 19.8\% [476]), which is consistent with our recruitment strategy. 


\section{Predicted probabilities of current rural practice, according to duration of rural background, by clinical school attended*}

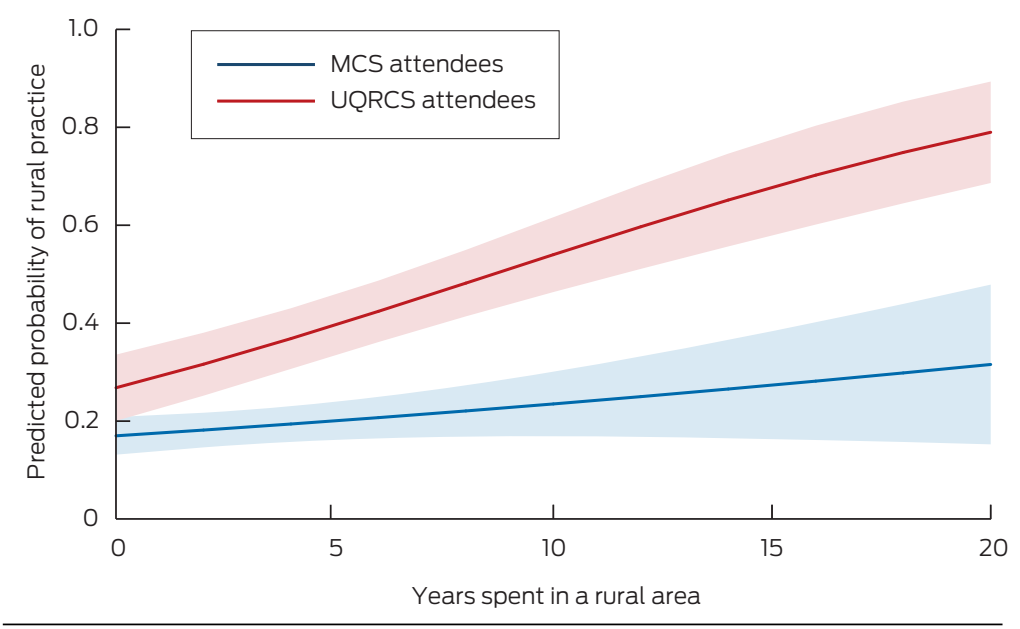

MCS = metropolitan clinical school. UQRCS = University of Queensland Rural Clinical School. * Based on a logistic regression model with the following as explanatory variables: UQRCS attendance $v$ MCS attendance, years spent in a rural area (since primary school and before commencing University of Queensland medical degree) as a continuous variable, and an interaction term between these two variables. Shaded bands represent $95 \% \mathrm{Cls}$.

\section{Discussion}

Our study shows that rural background and 1 or 2 years of UQRCS training are independent predictors of subsequent rural practice after adjusting for confounders including partnership status, partner's rural background and bonded scholarship. For UQRCS attendees, a positive, nearly linear, correlation exists between the probability of rural practice and duration of rural background over a range of $0-20$ years. In addition, there was a similar beneficial effect across inner regional, outer regional, remote and very remote areas on rural practice.

It also shows that there is a strong positive interaction between rural background and UQRCS attendance in enhancing the probability of rural practice. In contrast, training students with a rural background at an MCS or training students with a metropolitan background at UQRCS does not have a statistically significant effect on probability of rural practice (although trends exist [ORs, 1.46-1.83]). Our finding that having a bonded scholarship is associated with rural practice is not unexpected given that these graduates are financially committed to working in a rural area or an underserviced area, many of which are rural.
Few studies have used multivariate analysis to identify independent determinants of rural practice. A retrospective survey of 264 rural and 179 urban practitioners in Ontario, Canada, found that rural background and any rural undergraduate training were independent predictors of rural practice (ORs, 3.31 and 2.46, respectively), as were rural postgraduate training, medical school location and being male. ${ }^{19}$ In this survey, the main effects were reported but the interactions were not.

An Australian survey of 268 rural and 236 metropolitan general practitioners found statistically significant associations between current rural practice and rural background, rural school education and partner with a rural background. ${ }^{20}$ In the multivariate analysis, only rural primary school and partner with a rural background were independently associated with rural practice (ORs, 2.43 and 3.14, respectively).

A multivariate logistic regression analysis with seven variables using a sample of 359 medical graduates in the US found that the only independent predictors of rural practice were rural background and intention at medical school entry to become a family physician..$^{18}$ Interaction between the variables was not reported.

At the University of Minnesota (UMN) Medical School, 27\% of students had one or two different rural exposures - first 2 years of undergraduate training in a regional city (UMN-Duluth) and/or 9 months with a primary care preceptor in a rural community in their third year (Rural Physician Associate Program [RPAP].${ }^{25}$ In multivariate analysis for the outcome of current rural practice, RPAP, UMN-Duluth and rural background were independent predictors of rural practice (ORs, 4.6, 4.1 and 2.8, respectively). Other confounders were not assessed. When two- and three-way interactions were assessed, only UMN-Duluth training plus rural background was statistically significant and the interaction was negative (OR, 0.56 [95\% CI, 0.33-0.96]), in contrast to our finding.

In a recent data-linkage study conducted by the Rural Clinical School of Western Australia (RCSWA), rural practice of 1017 medical graduates who graduated during the period 2002-2009 was determined from the AHPRA register and rural background was defined by medical school entry through a quarantined rural pathway. ${ }^{26}$ In a multivariate model with interactions, the OR for rural practice for students with rural background who attended RCSWA compared with those with metropolitan background who did not attend RCSWA was 7.5 (95\% CI, 3.5-15.8) - a very similar result to ours.

Although the study did adjust for age and sex, no adjustments were made for other confounders. Further, the proportion of graduates practising rurally in the Western Australian and Queensland cohorts is markedly different $(7.7 \%$ and $27.2 \%$, respectively), which may be partly due to the lower proportion of the population living, over the study period, in areas categorised as RA2 to RA5 in Western Australia compared with Queensland ( $27 \%$ v $42 \%)$.

Our study has strengths and limitations. The main strength is that extensive data were available on sufficient numbers of graduates with different exposures to provide 
power for multivariate analyses with interactions on the outcome of rural practice. The study was conducted in a single large medical school with a uniform curriculum across 4 years, except that in year 3 and/or year 4 clinical training was delivered at UQRCS or an MCS. The findings may be generalisable to other Australian medical schools that offer MCS and RCS placements but perhaps not to regional medical schools that have much higher proportions of rural background students and routinely deliver rural exposure across multiple years of their courses. ${ }^{27}$

Our sample represents only $30.4 \%$ of domestic 2002-2011 medical graduates from UQ, so there could have been participation bias. However, a previous study with a response rate of $64 \%$ found that $40 \%$ of UQRCS attendees were in rural practice ${ }^{28}$ a very similar result to ours (42\%). In addition, the consistency of the result for rural practice between 2007-2011 graduates and 2002-2006 graduates (Appendix 2) suggests no participation bias relating to time since graduation. Further, our analysis of representativeness suggests that sampling would not have caused significant bias in the results.

Our results may be affected by self-selection bias regarding clinical school attended - that is, students who attend UQRCS may do so because they intend to enter rural practice. In the first few years of teaching at UQRCS, most students were conscripted, but in recent years $70 \%-90 \%$ have had UQRCS placement as their first preference. However, other factors influence students' first preferences, such as free or highly subsidised accommodation, academic reputation, patient and teacher access, lifestyle and work opportunities. ${ }^{24,29}$ Rural intention has been shown to be associated with other rural exposures (rural background or rural upbringing, having a spouse who had lived in a rural area), ${ }^{30,31}$ which we adjusted for. Also, other unknown confounders may have influenced our results.

Between 2002 and 2012, the number of full-time equivalent doctors practising in Australia per 100000 population (FTE rate) increased by $33 \%$ in metropolitan areas but increased by $50 \%-75 \%$ in rural areas, ${ }^{32,33}$ indicating reversal of the previous downward trend seen for GPs. ${ }^{1}$ Nevertheless, the FTE rate remains 33\%-39\% lower in rural areas for all doctors and $48 \%-$ $78 \%$ lower for specialists, so more needs to be done.

Rural clinical placements are limited and more expensive than metropolitan clinical placements, so policy measures that maximise the costeffectiveness of RCS programs are warranted. Our results suggest that the probability of rural practice could be increased by policies that increase the proportion of RCS attendees who have a rural background and who attend for more than one year. In addition, preferential recruitment of students with a background of longer-term rural residence should be considered.

Acknowledgements: This study was funded by the Australian Government Department of Health and Ageing under the Rural Clinical Training and Support program.

Competing interests: No relevant disclosures.

Received 20 Feb 2014, accepted 08 Aug 2014. 
1 Johnston G, Wilkinson D. Increasingly inequitable distribution of general practitioners in Australia, 198696. Aust N Z J Public Health 2001; 25: 66-70.

2 Last JM. Medical manpower in Australia. Med J Aust 1964; 1: 244-252.

3 Powell OW, Stabler PJ. Medical manpower in Queensland. Brisbane: Health Services Planning and Development Unit, Department of Health, 1976.

4 Scotton RB. Medical manpower in Australia. Med J Aust 1967; 1: 984-990.

5 Brooks RG, Walsh M, Mardon RE, et al. The roles of nature and nurture in the recruitment and retention of primary care physicians in rural areas: a review of the literature. Acad Med 2002; 77: 790-798.

6 Colditz GA, Elliott CJ. Queensland's rural practitioners: background and motivations. Med J Aust 1978; 2: 63-66.

7 Cullison S, Reid C, Colwill JM. Medical school admissions, specialty selection, and distribution of physicians. JAMA 1976; 235: 502-505

8 Kamien M, Buttfield IH. Some solutions to the shortage of general practitioners in rural Australia. Part 1. Medical school selection. Med J Aust 1990; 153: 105-107.

9 Kamien M, Buttfield IH. Some solutions to the shortage of general practitioners in rural Australia. Part 2. Undergraduate education. Med J Aust 1990; 153: 107-108, 112.

10 Kamien M, Buttfield IH. Some solutions to the shortage of general practitioners in rural Australia. Part 3. Vocational training. Med J Aust 1990; 153: 112-114.

11 Kamien M, Buttfield IH. Some solutions to the shortage of general practitioners in rural Australia. Part 4. Professional, social and economic satisfaction. Med J Aust 1990; 153: 168-171.

12 Laven GA, Beilby JJ, Wilkinson D, McElroy HJ. Factors associated with rural practice among Australian-trained general practitioners. Med J Aust 2003; 179: 75-79.
13 Rabinowitz HK. Relationship between US medical school admission policy and graduates entering family practice. Fam Pract 1988; 5: 142-144.

14 Reid P, Smith L. Country doctors in Western Australia. Aust Fam Physician 1976; 5: 6-8.

15 Rolfe IE, Pearson SA, O'Connell DL, Dickinson JA. Finding solutions to the rural doctor shortage: the roles of selection versus undergraduate medical education at Newcastle. Aust N Z J Med 1995; 25: 512-517.

16 Wilkinson D, Laven G, Pratt N, Beilby J. Impact of undergraduate and postgraduate rural training, and medical school entry criteria on rural practice among Australian general practitioners: national study of 2414 doctors. Med Educ 2003; 37: 809-814.

17 Ranmuthugala G, Humphreys J, Solarsh B, et al. Where is the evidence that rural exposure increases uptake of rural medical practice? Aust J Rural Health 2007; 15: 285-288.

18 Rabinowitz HK, Diamond JJ, Hojat M, Hazelwood CE. Demographic, educational and economic factors related to recruitment and retention of physicians in rural Pennsylvania. J Rural Health 1999; 15: 212-218.

19 Rourke JTB, Incitti F, Rourke LL, Kennard M. Relationship between practice location of Ontario family physicians and their rural background or amount of rural medical education experience. Can J Rural Med 2005; 10: 231-240.

20 Wilkinson D, Beilby JJ, Thompson DJ, et al. Associations between rural background and where South Australian general practitioners work. Med J Aust 2000; 173: 137-140.

21 Brookes J. Australia develops national strategy for bringing physicians to rural areas CMA J 1994; 150: 576 578.

22 Norington M. An update on rural general practice education initiatives to meet rural workforce needs: Progress and recent developments. Aust J Rural Health 1997; 5: 204-208.

23 Australian Bureau of Statistics. Australian Standard Geographical Classification. Canberra: ABS, 2011. http:// www.ausstats.abs.gov.au/ausstats/subscriber.nsf/0/32
FBEDEIEA4C5800CA25791F000F2EIC/\$File/att98dqt pdf (accessed Feb 2014).

24 Humphreys JS, Prideaux D, Beilby JJ, Glasgow NJ. From medical school to medical practice: a national tracking system to underpin planning for a sustainable medical workforce in Australasia. Med J Aust 2009; 191: 244-245.

25 Zink T, Center B, Finstad D, et al. Efforts to graduate more primary care physicians and physicians who will practice in rural areas: examining outcomes from the University of Minnesota Duluth and the rural physician associate program. Acad Med 2010; 85: 599-604.

26 Playford DE, Evans SF, Atkinson DN, et al. Impact of the Rural Clinical School of Western Australia on work location of medical graduates. Med J Aust 2014; 200: 104-107.

27 Sen Gupta TK, Manahan DL, Lennox DR, Taylor NL. The Queensland Health Rural Generalist Pathway: providing a medical workforce for the bush. Rural Remote Health 2013; 13: 2319

28 Eley DS, Synnott R, Baker PG, Chater AB. A decade of Australian Rural Clinical School graduates - where are they and why? Rural Remote Health 2012;13: 1-10.

29 Krahe LM, McColl AR, Pallant JF, et al. A multi-university study of which factors medical students consider when deciding to attend a rural clinical school in Australia. Rural Remote Health 2010; 10: 1477.

30 Royston PJ, Mathieson K, Leafman J, Ojan-Sheehan 0. Medical student characteristics predictive of intent for rural practice. Rural Remote Health 2012; 12: 2107.

31 Jones M, Humphreys J, Prideaux D. Predicting medical students' rural practice intentions using data from the Medical Schools' Outcome Database. 10th National Rural Health Conference; 2009 May 17-20; Cairns, Australia.

32 Australian Institute of Health and Welfare. Medical workforce 2012. Canberra: AlHW, 2014. (AlHW Cat. No. HWL 54; National Health Workforce Series No. 8.)

33 Australian Institute of Health and Welfare. Medical labour force 2004. Canberra: AlHW, 2006. (AlHW Cat. No. HWL 39; National Health Labour Force Series No. 38.) 